\title{
Clinical study on electro acupuncture plus interferential current therapy for chronic fatigue syndrome
}

\begin{abstract}
Objective: To observe the clinical efficacy of electroacupuncture plus interferential current therapy (ICT) in treating chronic fatigue syndrome (CFS), and to explore the mechanism.

Research design and methods: Sixty eligible CFS patients were randomized into a treatment group and a control group, 30 in each group. The treatment group was intervened by electro acupuncture plus ICT, while the control group was by electroacupuncture alone. The therapeutic efficacies were evaluated after 2 treatment courses and compared between the two groups.

Results: The recovery rate and total effective rate were respectively $43.3 \%$ and $93.3 \%$ in the treatment group, versus $20.0 \%$ and $80.0 \%$ in the control group. There were significant differences in comparing the recovery rate and total effective rate between the two groups ( $\mathrm{P} \square 0.05$ ), showing that the recovery rate and total effective rate in the treatment group were higher than those in the control group.
\end{abstract}

Conclusion: Electroacupuncture plus ICT can produce a marked efficacy in treating CFS.

Keywords: acupuncture therapy, electroacupuncture, interferential current therapy, chronic, fatigue syndrome
Volume 4 Issue 4 - 2016

\author{
Xiong Chen,' Jia Liu,' XiWang,' Fang Huang,' \\ Jingping $\mathrm{Mu}^{\mathrm{I}}$ \\ 'Department of Chronic Disease and Sleep Disorder, Tai He \\ Hospital, College of Medicine, Xi'an Jiaotong University, China \\ ${ }^{2}$ Department of Psychiatric, Tai He Hospital, College of \\ Medicine, Xi'an Jiaotong University, China
}

Correspondence: Jingping Mu, Department of Chronic Disease and Sleep Disorder, Tai He Hospital, College of Medicine, Xi'an Jiaotong University, No.32 South MingRen Road Shiyan 442000, China, Emai dachangxiong@163.com

Received: April 27, 2015 | Published: November 15, 2016
Abbreviations: CFS, chronic fatigue syndrome; ICT, interferential current therapy; TCM, traditional chinese medicine;

\section{Introduction}

Chronic fatigue syndrome (CFS) is a disorder that causes extreme fatigue but without a clear reason, accompanied by impaired memory, sleep disorders, poor concentration, headache, muscle and joint aches, tender lymph nodes in neck or armpit, low fever, a sore throat, and postexertion malaise, as well as mental symptoms lasting at least 6 months, in absence of abnormal findings in physical or lab examinations. ${ }^{1}$ With the increase of rhythm of life and stress brought by competition, the prevalence rate of CFS has also been increasing. The persistent fatigue and pain seriously affect the physical and mental health of people, and thus, CFS has attracted wide attention in the recent years. ${ }^{2}$ According to the statistics, the incidence rate has approached $10 \%$ $20 \%$ in white-collar workers. ${ }^{3}$ Currently, the pathogenesis of CFS is undefined, and there is no specific treatment protocol brought up yet. Acupuncture-moxibustion therapy has been broadly applied in the treatment of CFS.$^{4-7}$ Recently, rehabilitation science has adopted interferential current therapy (ICT) in treating CFS and has achieved accurate therapeutic efficacy. We used electroacupuncture and ICT in the treatment of CFS, and the report is given as follows.

\section{Materials and methods}

\section{Diagnostic criteria}

It's made by referring to the diagnostic criteria of CFS stipulated by the Centers for Disease Control and Prevention (CDC). ${ }^{8}$ The individual has unexplained persistent or recurrent devastating fatigue lasting over 6months that is not relieved after sufficient rest, with the level of daily activities dropped by $50 \%$; accompanied by 4 or more of the following symptoms: fatigue lasting over 6months; persistent post-exertion malaise; sleep disorders; muscle aches; pharyngolaryngitis; impairment of memory or concentration; pain in the joints without swelling or redness; tender lymph nodes in the neck or armpit; headaches of a new type, pattern, or severity.

\section{Inclusion criteria}

Conforming to the diagnostic criteria; age 18-65years; denial of allergic constitution; in absence of contraindications of electro acupuncture and ICT; willing to participate in the study and having signed the informed consent form.

\section{Exclusion criteria}

Against the diagnostic criteria or inclusion criteria; chronic fatigue due to primary diseases, e.g. adverse reactions of medications, hypothyroidism, chronic hepatitis; age below 18 or over 65; pregnant women or women during lactation; other severe diseases, such as impairment of heart, lung, liver, or kidney, or psychosis.

\section{General data}

Sixty eligible CFS patients were either the outpatients or inpatients from our hospital recruited from January 2011 to September 2013 after they agreed to sign the informed consent form and finish the whole treatment protocol and evaluation of therapeutic efficacy. The 60 subjects were coded by their visiting order and then divided into a treatment group and a control group by the random number table, 30 in each group. There were no significant differences in comparing the gender, age, and average disease duration ( $\mathrm{P} \square 0.05$ ), indicating the comparability (Table 1).

\section{Treatment methods}

\section{Treatment group}

Electroacupuncture: Acupoints: Baihui (GV 20), Guanyuan (CV 4), Qihai (CV 3), and bilateral. 
Xinshu (BL 15), Ganshu (BL 18), Feishu (BL 13), Pishu (BL 20), Shenshu (BL 23), Neiguan (PC 6), Shenmen (HT 7), Sanyinjiao (SP 6), and Zusanli (ST 36). ${ }^{9-11}$

Table I Comparison of general data

\begin{tabular}{llllll}
\hline & \multicolumn{2}{c}{ Gender (case) } & Average age & Average duration \\
\hline Group & $n$ & Male & Female & $(x \pm \mathrm{s}$, year $)$ & $(x \pm \mathrm{s}$, month) \\
Treatment & 30 & 13 & 17 & $43.00 \pm 12.00$ & $16.5 \pm 7.8$ \\
Control & 30 & 14 & 16 & $41.00 \pm 13.00$ & $15.9 \pm 8$. \\
\hline
\end{tabular}

Operation: Each time, 5-7 of the above points were selected. After standard sterilization, filiform needles of $0.3 \mathrm{~mm}$ in diameter and $40 \mathrm{~mm}$ in length were used to perpendicularly puncture by depth of 1.0-1.3cun. When needling qi arrived, moderate reinforcingreducing needling techniques were applied and the needles were retained for $20 \mathrm{~min}$. Afterwards, 4-6 points were chosen to connect to electroacupuncture apparatus (G-6805, manufactured by Suzhou Hwato Medical Instrument Co. Ltd.), with sparse-dense wave and tolerable intensity. The treatment was given once a day, 10 sessions as a treatment course, for totally 2 courses, with $1 \mathrm{~d}$ interval between two courses.

ICT: The stereo-dynamic interferential electrotherapy machine (LDG-2, imported from Japan) was adopted, frequency $5 \mathrm{kHz}$, with two groups of round electrodes $(4 \mathrm{~cm} \times 4 \mathrm{~cm}$, constituted by 3 conductive rubbers). One group of electrodes was applied to bilateral trapezius muscles, and the other group was to the spine between Xinshu (BL 15) and Shenshu (BL 23). The parameters were as follows: medium frequency $(5000 \pm 200) \mathrm{Hz}$, alternating frequency 0-200 Hz, dynamic rhythm $(10 \pm 2) \mathrm{s}$, output current intensity beyond the sensation threshold (with obvious 'tingling' or 'pins and needles' sensation). ICT was given $30 \mathrm{~min}$ each time, 10 sessions as a treatment course, for totally 2 successive courses. The patients were asked to have sufficient bed rest and avoid over-exertion.

\section{Control group}

The control group was only given the same electroacupuncture treatment as mentioned above.

\section{Therapeutic efficacy}

\section{Criteria of therapeutic efficacy ${ }^{12}$}

Recovery: The major symptoms and complications were completely gone, and the patient can adapt to normal social life and work.

Markedly effective: The major symptoms and complications were relieved by $\geq 2 / 3$. Effective: The major symptoms and complications were relieved by $1 / 3-2 / 3$.

Invalid: The major symptoms and complications were relieved by $\square 1 / 3$, or there was no improvement.

\section{Statistical analysis}

The SPSS 19.0 statistics software was used. The measurement data were expressed as $(\mathrm{x} \pm \mathrm{s})$ and analyzed by $\mathrm{t}$-test; Chi-square test was used for the comparison of ratio. $\mathrm{P} \square 0.05$ was considered to indicate a statistical significance.

\section{Treatment results}

After 2 treatment courses, the recovery rate was $43.3 \%$ and total effective rate was $93.3 \%$ in the treatment group, versus $20.0 \%$ and $80.0 \%$ in the control group. The inter-group comparisons of recovery rate and total effective rate both showed statistically significant differences (both $\mathrm{P} \square 0.05$ ). It indicated that electro acupuncture plus ICT can produce a higher therapeutic efficacy than electroacupuncture alone (Table 2).

Table 2 Comparison of therapeutic efficacies between the two groups

\begin{tabular}{llllllll}
\hline Group & $\boldsymbol{n}$ & Recovery & Markedly Effective & Improved & Invalid & Recovery Rate (\%) & Total Effective rate (\%) \\
\hline Treatment & 30 & 13 & 7 & 7 & 8 & 2 & 43.31 ) \\
Control & 30 & 6 & II & 6 & 20 & 93.31 ) \\
\hline
\end{tabular}

\section{Discussion}

The pathogenesis and etiology of CFS are complicated. According to its symptoms, it should fall under the scope of 'Xu Lao' (deficient fatigue) in traditional Chinese medicine (TCM). ${ }^{13}$ The causes majorly are impairment due to excessive emotion, physical or mental overexertion, excessive sexual activities, improper diet, and congenital insufficiency. The etiology majorly involves the consumption of qi and blood, yin and yang, and the five Zang organs are affected. Currently, it's believed that the liver, spleen, and kidney are mainly affected, especially the liver and spleen, characterized by deficiency at the root but excess at the superficial. The deficiency will lead to stasis and blockage of qi and blood in meridians and collaterals. ${ }^{14}$ In modern medicine, the pathogenesis and etiology of CFS are not clearly defined yet, ${ }^{15}$ though infection, sleep condition, emotional stimulation, and genetic factor are believed to be involved. The abnormal alterations of these factors can lead to the changes of neuro endocrine and immune systems, subsequently causing CFS. ${ }^{15}$ Therefore, it's meaningful to explore the pathological mechanism and effective treatments from the perspective of TCM.

Modern medicine discovers that acupuncture can reflectively lead to the reaction of central nervous system, manifested by the regulation of fatigue. ${ }^{16}$ Located on the first lateral line of the Bladder Meridian on the back, the Back-Shu points of the five Zang organs are where the visceral qi pours into the back, so these points are used to treat diseases of Zang-fu organs. Besides, they also connect with the brain. Moreover, the Foot Taiyang governs the yang qi of the surface of body. Acupuncture at the Back-Shu points act to boost yang qi, and can treat the deficiency of the five Zang organs. ${ }^{11}$ As the Yuan-Primary point of the Heart Meridian, Shenmen (HT 7) works to calm the mind, and maintain the normal heart rate. Neiguan (PC 6) is the Luo-Connecting point of the Pericardium Meridian and crosses the Yin Link Vessel. It can smoothen the flow of thoracic qi, keep calmness, and harmonize the stomach. As the joint point of the three foot yin meridians and the Conception Vessel, Guanyuan (CV 4) is closely related to the primary qi, acting to warm and reinforce qi, and supplement the three foot yin meridians. Zusanli (ST 36) is used to supplement spleen, relieve fatigue, and improve the sleep quality. ${ }^{17}$ The yin essence is the basic element for the activities of human body, and normal living activities depend on the promotion of yang qi. Thus, the current study adopted electroacupuncture at Back-Shu points to supplement the five Zang organs, dredge the meridians and collaterals, and boost yang qi. 
ICT originated from the early 1950s. Bearing the advantages of both low frequency and medium frequency currents, ICT can produces its highest intensity inside of the body at where the currents cross, and the stimulation is deep and wide. The action mechanisms of ICT are majorly present on the following aspects. ${ }^{18}$ First, it produces content analgesic effect by inhibiting the sensory nerves and enhancing the pain threshold. It's reported that ICT can increase the pain threshold of the whole body when acting on lumbosacral region, which is possibly caused by the continuous dilation of capillaries and small arteries led by the stimulation of ICT to the endorphin system. Second, ICT opens topical capillaries by increasing the tension of smooth muscle. The dilation of arteries can improve the blood circulation and cause the contraction of skeletal muscles, which consequently prevent and treat the atrophy of muscles. Third, through acting on the autonomic nervous system and the organelles which are in charge of metabolisms, ICT can promote the topical blood circulation, and the absorption of inflammatory products, edema, and hematoma. Fourth, ICT can modulate the visceral functions. The action of ICT is deep enough to stimulate the autonomic nerves, improve the blood circulation of internal organs, and enhance the tension of gastrointestinal smooth muscles. Some scholars applied ICT to the stellate ganglion of the hypertension patients, and found that the systolic and diastolic pressures dropped. It's also found that ICT can increase the skin temperature of lower limbs, promote the blood circulation, and improve the lameness of patients with endarteritis obliterans when acting on lumbar sympathetic ganglia

With a high incidence rate in the whole world, CFS has gradually become a serious health problem and thus has drawn great attention from the relevant fields. At present, China is threatened by excessive fatigue. Therefore, the early prevention, diagnosis, and treatment of CFS should be an urgent subject of the modern medicine. Acupuncture-moxibustion plus ICT is effective in regulating the visceral function, improving immunity, and anti-fatigue, superior to single electroacupuncture method. Considering its high efficacy and short treatment course, this painless method is ideal for treatment of CFS.

\section{Acknowledgments}

None.

\section{Conflicts of interest}

Author declares there are no conflicts of interest.

\section{Funding}

None.

\section{References}

1. Lan CH, Feng LM, Yang S, et al. Comparison of clinical efficacy of treating CFS by moxibustion with acupuncture five internal organs Back-Shu points. Zhongguo Linchuang Yanjiu. 2012;4(18):49-51.
2. Mao M, Mou X, Chen X,et al. Clinical observation of 45 cases of chronic fatigue syndrome treated with acupuncture. Chengdu Zhongyiyao Daxue Xuebao. 2013:36(1):80-82.

3. Zeng R, Tang JL. Observations on the efficacy of thunder- fire moxibustion plus massotherapy in treating chronic fatigue syndrome. Shanghai Zhenjiu Zazhi. 2009;28(8):445-446.

4. Chen XH, Yang J, Sun W, et al. Clinical observation on acupuncture therapy for chronic fatigue syndrome. $J$ Acupunct Tuina Sci. 2012;10(5):292-295.

5. Ding DM, Li SK. Therapeutic observation on acupuncture at Huatuo Jiaji (EX-B 2) for chronic fatigue syndrome. Shanghai Zhenjiu Zazhi. 2012;31(10):716-718.

6. Li CX, Zhang W. Treatment of 22 cases of chronic fatigue syndrome by puncturing Back-Shu Points. J Acupunct Tuina Sci. 2010;8(6):368-370.

7. Wang Y, Xiao W, Wang J. Therapeutic observation on thunder-fire moxibustion for chronic fatigue syndrome. Shanghai Zhenjiu Zazhi. 2012;32(10):827-828.

8. Fukuda K, Straus SE, Hickie I, , et al. The chronic fatigue syndrome: a comprehensive approach to its definition and study. International chronic fatigue syndrome study group. Ann Intern Med . 1994;121(12):953-959.

9. Liu YL, Yang JJ, Yang LJ. Therapeutic effect of acupuncture of BackShu points treating chronic fatigue syndrome and effect of psychological state. Zhonghua Zhongyiyao Xuekan. 2013;31(10):2327-2329.

10. Yuan WL, Zhang LP, Yan YG, et al.Clinical observation of using acupuncture and moxibustion in the treatment of chronic fatigue syndrome. Zhongyi Xuebao. 2011;26(2):240-241.

11. Xiang H. Efficacy observation of acupuncture and massage on treating chronic fatigue syndrome. Zhongyi Linchuang Yanjiu. 2013;5(4):39-41.

12. Liu Q. Chinese medicine in treating chronic fatigue syndrome. Riben Yixue Jieshao. 1993;14(5):242.

13. Chen M, Zhou YS, He WL, et al. Therapeutic observation on acupuncture plus tuina for chronic fatigue syndrome. Shanghai Zhenjiu Zazhi. 2013;32(5):354-357.

14. Yao RM. Research ideas and methods of chronic fatigue syndrome in acupuncture clinic. Zhongyi Zazhi. 2006;4(4):303-304

15. Zhang CZ, Wang TF, Kong JH, et al. Case-control study on the pathogenic factors of chronic fatigue syndrome. Beijing Zhongyiyao Daxue Xuebao. 2004;27(2):33-37.

16. Zhao JM, Zhang S, Wang WY. Study on action of acupuncture against sports physio-psychologic fatigue. Zhongguo Zhenjiu. 2004;24(8):519.

17. Sun ZW, Fang MX, Liu HR. Clinical observation of therapeutic effect on treatment of chronic fatigue syndrome with $\mathrm{Ci}$ Xue Tong Jing method. Liaoning Zhongyi Zazhi. 2010;37(10):2022-2023.

18. Yan TB. Physiotherapy. Beijing: People's Medical Publishing House; 2008. p. 256-360. 